\title{
A COSMOLOGICAL WINDOW ON TRANS-PLANCKIAN PHYSICS
}

\author{
JÉRÔME MARTIN \\ Institut d'Astrophysique de Paris CNRS, \\ 98 boulevard Arago 75014 Paris, France \\ E-mail: jmartin@iap.fr \\ ROBERT H. BRANDENBERGER \\ Department of Physics, Brown University, \\ Providence, RI 02912, USA \\ E-mail: rhb@het.brown.edu
}

\begin{abstract}
In the framework of inflation, scales which nowadays correspond to large scale structures were smaller than the Planck length at the beginning of inflation. Therefore, measurements of CMBR anisotropy or surveys of galaxies and of clusters of galaxies could help us to probe physics beyond the Planck scale. In these proceedings, we study how the inflationary observables depend on trans-Planckian physics. It is found that there exist cases where adiabaticity can be violated and the primordial spectrum of the quantum cosmological perturbations modified. These cases correspond to rather drastic alterations of the dispersion relation. There exist also modifications of the standard physics which leave the power spectrum unchanged and therefore which are compatible with the data. In this sense, the presently available observations already constrain trans-Planckian physics.
\end{abstract}

Since the recent observations of the Cosmic Microwave Background Radiation (CMBR) anisotropy and the detection of the first acoustic peak at an angular scale of $\simeq 1^{0}$ by the BOOMERanG and MAXIMA-1 experiments 4 , it has become likely that inflation is the correct theory of the very early Universe. If so, this opens the interesting possibility to test some aspects of the physics beyond the Planck scale. Indeed, in most of the realizations of the idea of inflation, the phase of accelerated expansion lasts so long that present cosmological scales were below the Planck length at the beginning of inflation. Therefore, any alterations of the standard physics at extremely small scales could have direct consequences on what we observe today. Among the observables which would be changed is the power spectrum of the primordial quantum fluctuations. The goal of these proceedings is to study under which conditions the power spectrum can be modified by a change of the physics below the Plancklength.

As first discussed in Refs. 3 . 1.6 , a similar problem exists in black hole physics. However, in this context, there exist deep thermodynamical reasons which render the derivation of the Hawking radiation robust to modifications of the transPlanckian physics. For inflation, these reasons no longer exist (we recall that the power spectrum is no longer a thermal one) and therefore it would be incorrect to believe that this question is just a trivial technical extension of what has already been done in the context of black hole physics.

Since a theory of quantum gravity is not available nowadays, the question arises as to which kind of modifications should be implemented in the theory. It was suggested by Unruh 3 that a generic way to do it is to consider non standard 
dispersion relations of the form $\omega=F(k)$ which reduce to the standard one for large scales, i.e. $F(k) \simeq k$ when $k$ is smaller than a cutoff $k_{\mathrm{C}}$ corresponding to the characteristic scale $l_{\mathrm{C}} \simeq 1 / k_{\mathrm{C}}$. $l_{\mathrm{C}}$ could be, for example, the Planck scale. A theory of quantum gravity should provide us with the shape of $F(k)$ for large values of $k$. In absence of such a theory, we will consider different ad hoc shapes for $F(k)$. In cosmology, the physical wave number $k$ is related to the comoving one $n$ by $k=n / a(\eta)$ where $a(\eta)$ is the scale factor. We see that, in the context of cosmology, we are led to introduce time-dependent dispersion relations, namely $\omega_{\text {phys }}=F[n / a(\eta)]$.

The choice of the initial state also needs to be discussed carefully. Since the dispersion relation is modified, when the wavelength of a mode is smaller than the characteristic length, the mode does not behave as a free wave and the usual vacuum state cannot be chosen. However, in Refs. $\mathrm{A}$, it was shown that a direct generalization of the vacuum can be found and consists in the state which minimizes the energy density initially. In these proceedings, all the results are established under the assumption that the initial state is this "minimizing energy state"

In order to know whether a change in the spectrum is expected, one has to compare the characteristic time scale of evolution of the physical frequency, $\left(1 / \omega_{\text {phys }}\right)\left(\mathrm{d} \omega_{\text {phys }} / \mathrm{d} \eta\right)$ with the Hubble time $\mathcal{H} \equiv a^{\prime} / a$ in the regime where the physical wavelength is smaller than the characteristic scale, $\lambda / l_{\mathrm{C}} \ll 1$. For this purpose, it is convenient to define an "adiabaticity coefficient" by

$$
\alpha(n, \eta) \equiv\left|\frac{\mathcal{H}}{\frac{1}{\omega_{\text {phys }}} \frac{\mathrm{d} \omega_{\text {phys }}}{\mathrm{d} \eta}}\right| .
$$

When $\alpha \gg 1$, the effective physical frequency can be considered as constant and the solution of the modified mode equation has time to adjust itself to track the standard solution. In this case, we have adiabaticity and no change is expected. On the contrary, if $\alpha \simeq 0$ adiabaticity is violated and some alterations of the standard power spectrum is likely to appear. In the standard case, we have $\alpha=1$ since the time scales of evolution of the physical frequency and of the Hubble time are the same.

In Refs. 国, we have considered the following dispersion relations

$$
\omega=k_{\mathrm{C}} \tanh ^{1 / p}\left[\left(\frac{k}{k_{\mathrm{C}}}\right)^{p}\right], \quad \omega=k \sqrt{1+b_{m}\left(\frac{k}{k_{\mathrm{C}}}\right)^{2 m}}
$$

which were first introduced in Refs. 3 . In the second of these equations, $m$ and $b_{m}$ are arbitrary numbers and $b_{m}$ can be either positive or negative. These dispersion relations are displayed in Fig. (1).

The adiabaticity coefficient can be computed exactly once a model of inflation is chosen. For simplicity, we consider power-law inflation where the scale factor is given by $a(\eta)=l_{0}|\eta|^{1+\beta}$, where $\beta \leq-2$. $\beta=-2$ corresponds to the De Sitter

${ }^{a}$ In Refs. 8 another initial state was considered. Of course, only the "minimizing energy state" is physical and the purpose of introducing this other state was just to test the dependence of the final result on the initial conditions.

mg9d: submitted to World Scientific on November 6, 2018 


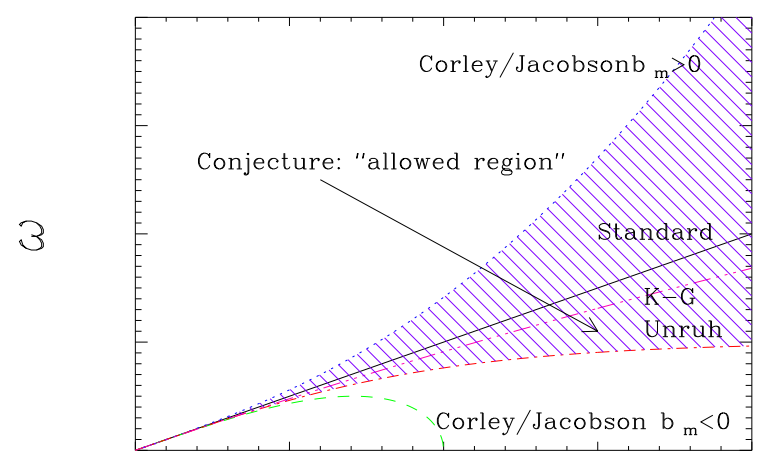

K

Figure 1. Different dispersion relations

spacetime. For the Unruh dispersion relation, one finds

$$
\alpha_{\mathrm{U}}(n, \eta)=\left(\frac{l_{\mathrm{C}}}{\lambda}\right)^{-p} \sinh \left[\left(\frac{l_{\mathrm{C}}}{\lambda}\right)^{p}\right] \cosh \left[\left(\frac{l_{\mathrm{C}}}{\lambda}\right)^{p}\right] .
$$

When $l_{\mathrm{C}} / \lambda \ll 1$, one has $\alpha_{\mathrm{U}}=1$. On the contrary, if $l_{\mathrm{C}} / \lambda \gg 1$ then $\alpha_{\mathrm{U}} \rightarrow \infty$ and adiabaticity is satisfied. Therefore, we expect no change for the Unruh dispersion relation. This is confirmed by the results of Refs. dispersion relation the result reads

$$
\alpha_{\mathrm{CJ}}(n, \eta)=\frac{1+b_{m}\left(l_{\mathrm{C}} / \lambda\right)^{2 m}}{1+\left(b_{m}+1\right)\left(l_{\mathrm{C}} / \lambda\right)^{2 m}} .
$$

One checks again that in the case where $l_{\mathrm{C}} / \lambda \ll 1$, one has $\alpha_{\mathrm{CJ}}=1$. If $l_{\mathrm{C}} / \lambda \gg 1$, the adiabaticity coefficient tends to the constant $1 /(m+1)<1$. If $b_{m}<0, \alpha_{\mathrm{CJ}}$ goes to zero at the point where $\omega=0$ and adiabaticity is violated. In addition, it blows up at the point where $\mathrm{d} \omega / \mathrm{d} \eta=0$. The evolution of the adiabaticity coefficients is displayed in Fig. (2).

The case in which a difference is obtained can be viewed as rather extreme. Indeed, when $b_{m}<0$, the dispersion relation becomes complex which causes no problem since this situation can even occur in classical physics. However, the initial state is chosen in this region. Since it is the state which minimizes the energy and since the energy might be unbounded from below in this region, this choice could be problematic.

This leads us to the idea that we should explore a case in which the dispersion relation lies between the standard relation and Unruh's one in Fig. (11). In this

mg9d: submitted to World Scientific on November 6, 2018 


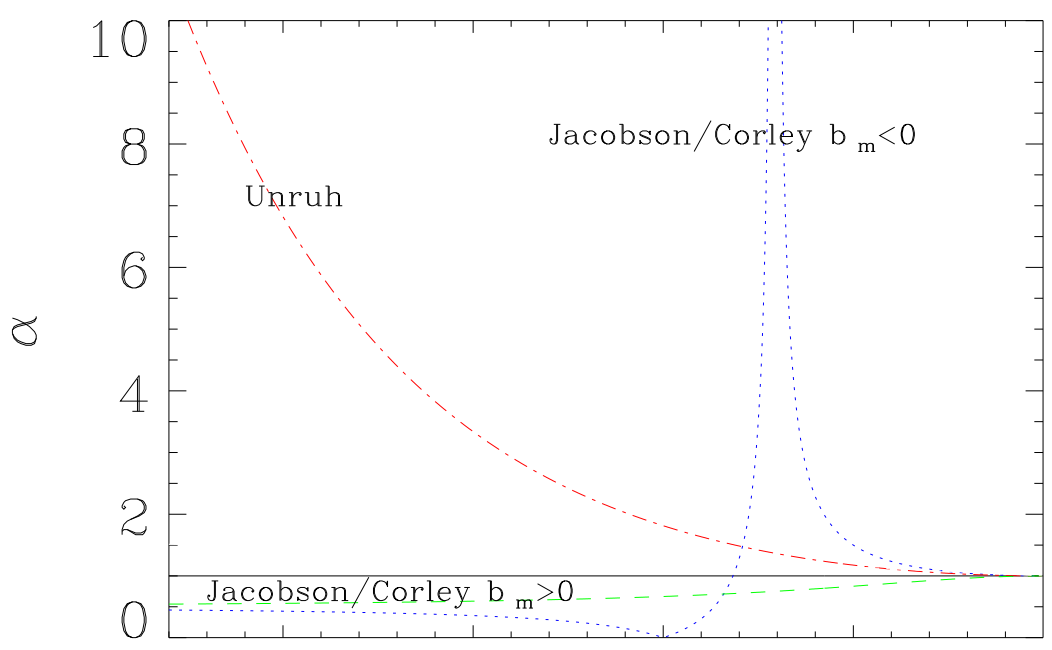

n

Figure 2. Different adiabaticity coefficients

case, the alteration of the dispersion relation does not have the two shortcomings mentioned before. Typically, such a relation is given by

$$
\omega=\frac{k_{\mathrm{C}}}{\gamma} \ln \left(1+\gamma \frac{k}{k_{\mathrm{C}}}\right),
$$

where $\gamma$ is a free parameter. Interestingly enough, the case $\gamma=1$ has already been studied by Kowalski-Glikman 1 for completely different reasons. The adiabaticity coefficient is given by

$$
\alpha_{\mathrm{KG}}(n, \eta)=\frac{\lambda}{\gamma l_{\mathrm{C}}}\left(1+\gamma \frac{l_{\mathrm{C}}}{\lambda}\right) \ln \left(1+\gamma \frac{l_{\mathrm{C}}}{\lambda}\right) .
$$

When $l_{\mathrm{C}} \gg \lambda, \alpha_{\mathrm{KG}}$ tends very slowly to infinity with a rate which depends on $\gamma$. Therefore, it seems that adiabaticity is preserved and that we should not expect any change in the final spectrum. This is also the result reached by the study done in Ref. 10 .

The conclusion of this work is mitigated. The predictions of inflation appear less robust than those of black hole physics. It is possible to find cases where adiabaticity is violated and where the medifications to the usual power spectrum can be explicitly calculated, see Refs. 6 . However, these modifications can be considered as very strong and each time one tries to introduce less drastic modifications, the final result is the same as for the unmodified dispersion relation. In a certain sense, the presently available data already constrains the physics beyond the Planck scale. Therefore, it is reasonable to conjecture that the allowed region of monotonic dispersion relations (the present study does not constrain oscillatory

mg9d: submitted to World Scientific on November 6, 2018 
dispersion relations) lies roughly between the standard dispersion relation and the Unruh dispersion relation, i.e. corresponds to the dashed region in Fig. (11). In fact, when the dispersion relation lies slightly lower than Unruh's relation, one should still have no modifications of the spectrum and therefore, the dashed region can be considered as a lower bound on the region for unmodified spectra.

\section{References}

1. P. de Bernardis et al, Nature (London) 404, 955 (2000).

2. S. Hanany et al, preprint astro-ph/0005123 (2000).

3. W. Unruh, Phys. Rev. D51, 2827 (1995).

4. R. Brout, S. Massar, R. Parentani and P. Spindel, Phys. Rev. D52, 4559 (1995).

5. N. Hambli and C. Burgess, Phys. Rev. D53, 5717 (1996)

6. S. Corley and T. Jacobson, Phys. Rev. D54, 1568 (1996);

S. Corley, Phys. Rev. D57, 6280 (1998).

7. J. Martin and R. H. Brandenberger, hep-th/0005209.

8. R. H. Brandenberger and J. Martin, astro-ph/0005432.

9. J. C. Niemeyer, astro-ph/0005533.

10. J. Kowalski-Glikman, astro-ph/0006250. 